\title{
¿Septo o Tabique? Análisis Histórico, Etimológico y Pro- puesta para Terminología Anatómica Internacional (TAI)
}

\author{
Septum or "Tabique"? Historical, Etymological and Proposal \\ Analysis for International Anatomical Terminology (TAI)
}

\author{
Francisco Pérez-Rojas ${ }^{1,2}$; Lukas A. Canales-Guzmán ${ }^{3}$; Catalina Chirino-Letelier ${ }^{3}$ \& Ricardo S. Puebla-Wuth ${ }^{4}$
}

PÉrez-RoJas, F.; CANAles-guZMán, L. A.; ChIRINO-Letelier, C. \& PUEbLA-WUTH, R. S. ¿Septo o Tabique? Análisis Histórico, Etimológico y Propuesta para Terminología Anatómica Internacional (TAI). Int. J. Morphol., 37(2):766-772, 2019.

RESUMEN: El interés creciente por conciliar una terminología anatómica común hace imperativo llegar a establecer un lenguaje universal a lo largo del tiempo y focalizar este objetivo en un trabajo sistemático, que permita definir y denominar adecuadamente las estructuras que conforman el cuerpo humano (Álvarez Guisbert \& Campohermoso Rodríguez, 2007). En las ciencias morfológicas, la TAI establece que los términos para nombrar las estructuras anatómicas, ocupan como idioma oficial el latín y términos comunes del idioma español derivados de una raíz latina. En este trabajo se hace notar que una serie de estructuras anatómicas que en español han sido asignadas con el nombre tabique, no se corresponden etimológicamente con la raíz latina de la palabra con que originalmente fueron nombradas: Septum. Evidenciamos que la palabra Septum se traduce al español literalmente como septo, mientras que, el vocablo tabique proviene de una raíz de origen árabe tasbík; y, por tanto, no es un término que cumpla con los lineamientos de la TAI. Aquí damos cuenta, de acuerdo a un análisis etimológico e histórico de ambas palabras, que estas se hicieron sinónimos a lo largo del tiempo en el idioma español. Aun así, respetando la TAI, el término tabique no es apropiado puesto que su raíz lingüística es árabe; y, por tanto, sugerimos cambiar la traducción de Septum por septo en las estructuras anatómicas de las que da cuenta esta investigación.

PALABRAS CLAVE: Terminologia Anatomica; Septo; Tabique.

\section{INTRODUCCIÓN}

Para las diversas ciencias que utilizan la anatomía, como las ciencias de la salud, es de suma importancia contar con términos precisos, claros y concisos acerca del nombramiento de estructuras corporales, tanto en el estado de salud como en la enfermedad (Whitmore, 1999). Esta denominación, se debe hacer siguiendo un patrón establecido y reconocido por todos, situación que es provista por la Terminología Anatómica Internacional (TAI) en la actualidad (Comité Federal sobre Terminología Anatómica \& Sociedad Anatómica Española, 2001).

La TAI, fue desarrollada por la Federación Internacional de Asociaciones de Anatomía y redactada en latín, debiendo ser traducida a otros idiomas vernáculos por los anatomistas que hacen uso de estos términos, teniendo en consideración el que los nombres de las estructuras debían poseer un valor informativo (Moya et al., 2017). La Nómina Anatómica, determina que: el idioma anatómico es el latín con traducción al idioma nativo; e, indica, la supresión de epónimos y nombres propios; así como, utilización de planos y ejes anatómicos. En Tokio, en el año 1975, se realizan enmiendas a las nóminas con algunas importantes conclusiones para esta investigación, tales como: el latín, es el idioma universal del estudio de las ciencias morfológicas; cada estructura, debe tener un solo nombre; los nombres son simples, breves y con valor informativo y descriptivo. Estas tres conclusiones son herramientas claves a la hora de investigar términos que, teniendo un único nombre en latín, han sido traducidos a idioma vernáculo con más de un término

\footnotetext{
${ }^{1}$ Profesor Asistente, Morfología Humana, Carrera de Medicina, Universidad Autónoma de Chile, Sede Talca, Chile.

${ }^{2}$ Magíster en Ciencias mención Morfología, Facultad de Medicina, Universidad de La Frontera, Temuco, Chile.

${ }^{3}$ Estudiante de Medicina, Universidad Autónoma de Chile, Talca, Chile.

${ }^{4}$ Docente Universidad de Los Lagos, Osorno, Chile.
} 
sinónimo (Tabla II). En el año 2011, se presentó la segunda edición de la TAI. La edición fue elaborada por el FIPAT, Programa Internacional Federativo en Terminologia Anatomica de la International Federation of Associations of Anatomists (IFAA) que reemplazó al Comité Federal sobre Terminología Anatómica (FCAT) (Federative International Programme on Anatomical Terminologies, 2011).

La versión en español de la TAI, fue elaborada gracias al trabajo conjunto de la IFAA y la Sociedad Anatómica Española (SAE), la cual fue publicada en el año 2001 y constituye la única edición en español de la TAI reconocida (Comité Federal sobre Terminología Anatómica \& Sociedad Anatómica Española).

La revisión de los orígenes históricos, que apoya el uso de la terminología, nos permite comprender la complejidad que existe en el establecimiento de una terminología anatómica común (Pérez-Rojas et al., 2018). Debido a la permanente y gran relevancia de la anatomía como herramienta o sustento de información, se genera continuamente el empleo de términos que no cumplen de manera estricta con lo estipulado por la TAI (Grkovic et al., 2009). Esto, se hace relevante cuando se traduce desde el latín al idioma vernáculo, lo que genera confusiones etimológico-conceptuales entre los diversos términos presentes en el idioma español (Vargas et al., 2016; Pérez-Rojas et al.). Además, en el Español, una lengua romance proveniente del latín vulgar, existen palabras con raíz en el árabe (Resnick, 1981), por lo que el análisis lingüístico de tipo etimológico-histórico de las palabras ocupadas en las traducciones presentes en la actual terminología anatómica adquiere una especial relevancia, cuando de traducción de términos anatómicos al español se trata. El interés creciente por conciliar un idioma común y apegado a las normas establecidas, hace imperativo focalizar un trabajo sistemático y continuo que permita definir y denominar adecuadamente las estructuras que conforman el cuerpo humano (Álvarez Guisbert \& Campohermoso Rodríguez). Debido a lo señalado anteriormente, se analiza en este trabajo el significado original del término Septum, advirtiendo acerca de su inconsistente traducción al español como Tabique, término de raíz lingüística árabe y que es ampliamente utilizado para designar una serie de estructuras anatómicas cuyo nombre de origen es Septum (Tabla I); pero, cuya, debida traducción al español debiera ser Septo, que es lingüísticamente la expresión de la raíz latina de Septum. Un análisis etimológico e histórico de los términos Septum, Septo y Tabique, permite proponer con evidencias robustas que, el cambio de nombre para los términos denominados como Tabique por la TAI, surgió del uso sinonímico dado a las palabras Septo y Tabique a lo largo del tiempo en la lengua española; y, sin embargo, constatamos que éstas surgen de raíces lingüísticas diferentes, siendo Septo la expresión que deriva realmente del término latino original Septum. Finalmente, se denota el uso en la TAI de más de un término para designar estructuras cuyo nombre original en latín es Septum (Tabla II), y proponemos se les asigne el término único de denominación Septo, respetando, por sobre todo, la raíz latina del término original

\section{MATERIAL Y MÉTODO}

Se realizó una revisión de la TAI en Español, del año 2001, para los términos incluidos en las Tablas I y II.

Se revisaron artículos científicos del área, para corroborar que los términos escogidos no hubiesen sido corregidos en otras publicaciones (PubMed, Cochrane y Scielo) en idioma español. Se desarrolló un análisis histórico y etimológico en diccionarios antiguos extraídos del Nuevo Tesoro Lexicográfico de la Real Academia Española de la Lengua (RAE); El diccionario médico-biológico, histórico y etimológico de Salamanca; los diccionarios de latín-español como el "Diccionario Vox", "Diccionario Latín" de la editorial SM, y la tercera edición del "Diccionario Ilustrado LATINO-ESPAÑOL de la editorial SPES. Además, para determinar la ubicación en el tiempo del error lexicográfico se utilizó el Atlas de Historia de España (García de Cortázar, 2005), de la Real Academia de la Historia.

\section{RESULTADOS}

Al hacer la revisión de la TAI, se encontró que los términos de la Tabla I tenían como nombre original asignado la palabra Septum, cuya traducción literal al español es: Septo; y, sin embargo, se encontraban traducidas al español con el vocablo: Tabique. Ahora bien, en el Diccionario de la RAE, se indica lo siguiente, respecto a Tabique y Septo:

Tabique: Del ár. hisp. tasbík, y este del ár. clás. tasbık 'acción de enrejar'. 1. m. Pared delgada que sirve para 
Tabla I. Traducciones de Septum al español.

\begin{tabular}{lll}
\hline $\begin{array}{l}\text { Código de } \\
\text { terminología }\end{array}$ & \multicolumn{1}{c}{ Raíz latina } & \multicolumn{1}{c}{ Español } \\
\hline A02.1.00.087 & Septum nasi osseum & Tabique nasal óseo \\
A02.1.03.031 & Septum sinuum frontalium & Tabique del seno frontal \\
A02.1.05.017 & Septum intersinu ale sphenoidale & Tabique intersinusal esfenoidal \\
A02.1.06.020 & Septum canalis musculotubarii & Tabique del conducto musculo tubárico \\
A04.6.03.006 & Septum intermuseulare brachi mediale & Tabique intermuscular medial del brazo \\
A04.6.03.007 & Septum intermuseulare brachi laterale & Tabique intermuscular lateral del brazo \\
A04.7.03.004 & Septum intermusculare femoris laterale & Tabique intermuscular femoral lateral \\
A04.7.03.005 & Septum intermusculare femoris mediale & Tabique intermuscular femoral medial \\
A04.7.03.007 & Septum intermusculare vastoadductorium & Tabique intermuscular vastoaductorio / tabique intermuscular anteromedial \\
A04.7.03.015 & Septum femorale & Tabique femoral \\
A04.7.03.022 & Septum intermusculare cruris anterius & Tabique intermuscular anterior de la pierna \\
A04.7.03.023 & Septum intermusculare cruris posterius & Tabique intermuscular posterior de la pierna \\
A05.1.04.024 & Septum linguae & Tabique lingual \\
A06.1.02.004 & Septum nasi & Tabique Nasal \\
A09.4.01.019 & Septum penis & Tabique \\
A09.4.03.004 & Septum scroti & Tabique escrotal \\
A14.1.01.311 & Septum cervicale intermedium & Tabique cervical intermedio \\
A14.1.02.009 & Septum medianum posterius & Tabique medio posterior: Tabique medio dorsal \\
\hline
\end{tabular}

Tabla II. Ejemplos de asignación de dos términos a una sola estructura anatómica.

\begin{tabular}{lll}
\hline Código de Terminología & Raíz latina & Español \\
\hline A12.1.00.013 & Septum interventriculare & Septo interventricular: Tabique interventricular \\
A12.1.00.016 & Septum atrioventriculare & Septo a trioventricular: Tabique atrioventricular \\
A12.1.00.019 & Septum interatriale & Septo interatrial: \\
& & Tabique interatrial \\
\hline
\end{tabular}

separar las piezas de la casa. 2. m. División plana y delgada que separa dos huecos. El tabique de las fosas nasales. 3. m. Méx. Ladrillo (\|masa rectangular de arcilla). Tabique de carga $1 . \mathrm{m}$. tabique que está hecho con ladrillos sentados de plano y sirve para cargar en él las vigas de una crujía. Tabique de panderete $1 . \mathrm{m}$. tabique que está hecho con ladrillos puestos de canto. Tabique sordo 1 . m. tabique que se compone de dos tabiques de panderete separados y paralelos.

Septo: Del lat. Saeptum 'seto, cercado, muro'.1. m. Zool. Tabique que divide de un modo completo o incompleto una cavidad o partes del cuerpo en un animal.

Es posible reconocer, que ambos términos presentan una raíz de distinto origen lingüístico, Tabique proviene del árabe hispánico tasbík, un término derivado del árabe clásico tasbık, y Septo proviene del latín Saeptum el cual, a su vez, es la raíz latina original de Septum (Diccionario Vox latín, 1968) (Fig.1).

Dadas las diferencias etimológicas encontradas, se analizaron los diccionarios históricos de la lengua española, para hallar el período histórico en el cual ambas palabras se hicieron sinónimos en el español, y comunes en el uso de este idioma. Siguiendo la metodología propuesta, al realizar la búsqueda en el diccio-

\section{septum, v. sceptum.}

Fig. 1. Diccionario Latín Vox.

nario web de la Universidad de Salamanca (Universidad de Salamanca, 2018) no se obtienen resultados. Seguidamente, se realizó una búsqueda etimológica de estos términos en: etimologias.dechile.net (Anders, 2019), para ampliar la diversidad de hallazgos, encontrándose, en relación a Tabique: Al igual que algunas otras palabras relacionadas con la construcción (v.g. acequia, albañil. alberca, alcantarilla, aljibe, azotea), la palabra tabique viene del árabe hispano y se refiere a una "pared delgada, construida con ladrillos que separa habitaciones"; y, de Septo: La palabra septo (membrana que separa dos cavidades, como en la nariz) viene del latín Septum, participio pasivo del verbo saepire (cercar).

Para reafirmar la evidencia etimológica encontrada, obtener fundamentos históricos y analizar la sinonimia de ambos términos, se recurrió a la potente herramienta histórica del español: el Nuevo Tesoro Lexicográfico de la RAE. Se consultaron diccionarios antiguos digitalizados desde el año 1495 hasta 1989, en los que se obtuvieron los siguientes resultados: Los términos Tabique (Figs. 2 a 7) y Septo (Figs. 8 a 14) se 
PÉREZ-ROJAS, F.; CANALES-GUZMÁN, L. A.; CHIRINO-LETEliER, C. \& PUEBLA-WUTH, R. S. ¿Septo o Tabique? Análisis Histórico, Etimológico y Propuesta para Terminología Anatómica Internacional (TAI). Int. J. Morphol., 37(2):766-772, 2019.

ocupaban en la antigüedad en contextos distintos. Se observa que desde el año 1611, en el diccionario Rosal, se manifiesta la connotación de la palabra Tabique enmarcada dentro del ámbito de la construcción (Sobrino en 1705; y Academia usual de 1780) (Figs. 3 y 4). Además, se evidencia en las definiciones, el material que constituye un tabique, repitiéndose en la gran mayoría de los diccionarios esta intención de construcción.

Por su parte, el término Septo presenta documentación desde el año 1495 hasta la actualidad, en la que se evidencia que este concepto se enmarca dentro de las estructuras divisorias por definición, pero no se observa una vinculación exclusiva con las áreas de albañilería y construcción (Figs 8 a 14). Ahora bien, dado que las apariciones del término Septo en los libros más antiguos son escasas, se realizó un análisis etimológico en el Diccionario de etimologías de la lengua castellana de Ramón Cabrera, encontrando que la palabra Seto que significa Cerco o Cerca, tiene una raíz latina que correspondería igualmente a Septum (Cabrera et al., 1837; y, Diccionario Nebrija de 1495). Las primeras interpretaciones encontradas para Seto fueron, Siepe que significa Cerca, y Tramezzamento que significa Seto (Diccionario Casas de 1570). Además, en 1611 ya aparecía con el significado de "cerca hecha en la viña, o huertas" (Covarrubias en 1611) (Fig. 9), Esto, da cuenta del porque los significados de ambos términos se hicieron equivalentes en el idioma, para ser interpretados como sinónimos lo cual queda en evidencia en múltiples diccionarios al definir Septo (Diccionario Alemany y Bolufer de 1917; y Academia usual de 1989) (Figs. 13 y 14).

\section{Tabique}

Diccionario Rosal 1611: es Arábigo, el cual le llama Texbiq, y de allí Taíbíque, y así Tabuco a la casa pobre, porque es de tabiques, y no de tapia, o piedra (Fig. 2).

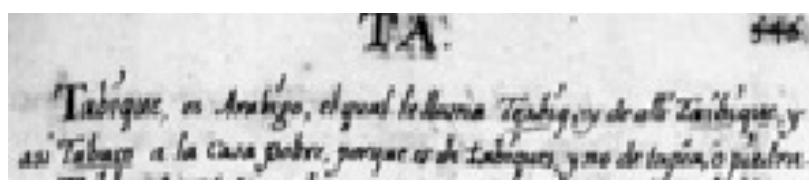

Fig. 2. Rosal 1611.

Diccionario Sobrino 1705: m, Pared de ladrillos, Cloifon de briques (Fig. 3).

\section{Tabique, m. pared de ladrillos, Chilon de briques.}

Fig. 3. Sobrino 1705.
Diccionario Academia Usual 1780: s. m. Pared delgada que se hace de cascotes, ó ladrillo, ó adobes puestos al canto, trabados con yeso. Comunmente sirve para la division de los quartos, ó aposentos de las casas. Crotitios puries, vel diplintbius (Fig. 4).

\section{TABIQUE s. na. Pared delendia que ie hace de

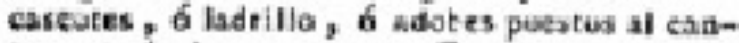

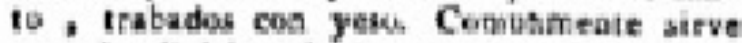

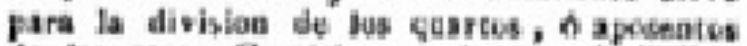

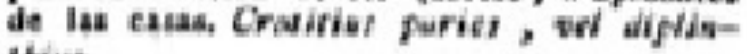 Ihish.}

Fig. 4. Academia Usual 1780.

Diccionario Domínguez 1853: s. m. Arq. Entramado de madera y fábrica que no llega a un pié de grueso.= Colgado; el que no sube desde la planta baja.= de eleccion; lo mismo que maestro.= de panderete; que está formado de ladrillos puesto de canto.= sordo; el que está construido con dos superficies de ladrillos verticales, quedando un hueco en medio (Fig. 5).

\section{Tablaque, s. m. Arq. Entramado de} madera y fábrica que no llezas á un pié de grueso.=Colgado: el gue no sube desde la planta baja.=ic eleccion: lo mismo que macstro. $=d e$ panderete: que está formado Jo ledrilies puestos de canto. = sorlo; el que está construido con dos supertieies de ladrillos vern licales, quedando us huece en medio.

Fig. 5. Dominguez 1853.

Diccionario Zerolo 1895: [Del ár. Taxbic, pared de ladrillo] m. 1. Pared delgada que se hace de cascotes, ladrillos o adobes puestos de canto y trabados con yeso. Comúnmente sirve para la división de los cuartos ó aposentos de las casas. "Procedía de otra pared en medio, y con quien alindaban otros flacos tabiques" (CÉSP).

- 2. Zool. Ternilla que separa en el cuerpo dos partes iguales entre sí, como la que divide las fosas nasales, llamada también ternilla de la nariz.

- 3. Bot. Película ó membrana más ó menos resistente que separa en las plantas partes que desempeñan unas mismas funciones ó que tienen un mismo objeto. TABIQUES orulares.

- 4.- rectovaginal. Tabique que separa del recto la cavidad de la vagina, y que está formado por la unión de los tejidos recto y vaginal. 
PÉREZ-ROJAS, F.; CANALES-GUZMÁN, L. A.; CHIRINO-LETEliER, C. \& PUEBLA-WUTH, R. S. ¿Septo o Tabique? Análisis Histórico, Etimológico y Propuesta para Terminología Anatómica Internacional (TAI). Int. J. Morphol., 37(2):766-772, 2019.

- 5.- rectovesical. Tabique que resulta de la aproximación y adherencia de los tejidos correspondientes de la vejiga y el recto (Fig. 6).

- Taurutik. (bel ár. tarbic, pared dela. Arillo.) m. 1. Pared delzada que se hace do cascotes, laifrillos o adober peestos de cante y trabailos con yeso, Comúnamente sirve para la division de los cuartos 6 aposentos do las easas. a l'rocedia de otra parnd en medio, y roo quien alinilaban unos flacos tebiguet. (GESP.)

$-2 . \div Z$ Zool. Temilla que separa en el cuerpo des partes iguales coiru sI, como la quedivide las foras nasales, Ilamada tambien teraills de la neriz.

- 3. + Rat. Petieula 6 memhrana mas 6 monos resiste ble que separa co las plantaspartes que desempenan vos. misnas funciones of que tienesi un nitsase oljeto. Tabtotes orsCarcs.

-4. 8 - rceforagiad. Tabique que sopara del meto in eavidail de la vagina, $y$ que esta formado per la valon de los tejidos recte $y$ ragianl.

-5. $t-$ reteresieat. Tabiqee que resulia

to la aproxinación y alherenein dé tos tejidos correnyondientes de la vejigra y el rocto.

Fig. 6. Zerolo 1895 .

Diccionario Academia usual 1925: Por extensión, división plana y delgada que separa dos huecos. Él tabique de las fosas nasales (Fig. 7).

cuartos 0 apobentós de las ensus. II 2, Pur exbeusion, division plana y" detedada que

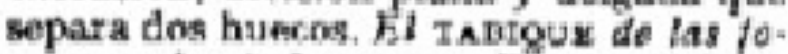

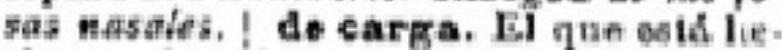

Fig. 7. Academia usual 1925 Seto (Septum).

Diccionario Nebrija 1495: seps.pis. Septum. 1. Sepimentum.1. (Fig. 8).

\section{Seto.feps.pie.feptumi.i.fepimentumt.t.}

Fig. 8. Nebrija 1495 .

Diccionario Covarrubias 1611: Lat, seps, sepis, septum.i. la cerca hecha en la viña, o huerta de cambroneras, zarzas, espinos, y otros arbustos que se cierran entre sí, e impiden la entrada (Fig. 9).

SET O, Lat:feps, lepis, feprum.i.la cerca hecha en la viñ a, o hueria de cam broner as, çarças, ef pinos, y otros arbuf: tos que le cierran entre fi, $\mathrm{e}$ impiden la entrada.

Fig. 9. Covarrubias 1611.
Diccionario Rosal 1611: El cercado, de septo, latino (Fig. 10).

\section{Seto, el corcado, de Jepto, Satino.}

Fig. 10. Rosal 1611.

\section{Septo}

Diccionario Bluteau 1721: id. on Tapigo (Traducción: Barricada) (Fig. 11).

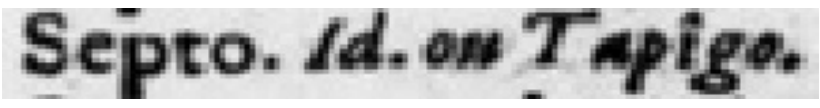

Fig. 11. Bluteau 1721.

Diccionario Academia Usual 1984: (Del latín Septum, p. p. de saepio, cercar, cerrar) m. Zool.. Tabique que divide de un modo completo o incompleto una cavidad o partes del cuerpo en un animal (Fig. 12).

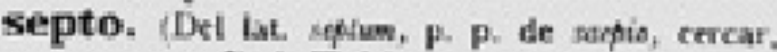
certar.) m. Zool. Tabique que divide de un modo completo a incompleto una cavidad a partes del cuerpo en un animal.

Fig. 12. Academia Usual 1984.

Diccionario de Alemany y Bolufer 1917: Septum (del lat. Septum, pared. Tabique). M. anat. Tabique membranoso o carnoso que separa dos cavidades (Fig. 13).

sEPTUM. (del lat. gentum, pared, tabique). m. Anat. Tabique membrinnoso 0 carnoso que bepara dos cavidades.

Fig. 13. Alemany y Bolufer 1917.

Diccionario Academia Usual 1989: Septo. m. Zool. Tabique que divide de un modo completo o incompleto una cavidad o partes del cuerpo en un animal (Fig. 14).

septo. m. Zaol. Tahique que divide de un modes completes o incompleio una cavidad o partes del euerpro en un animal.

Fig. 14. Academia Usual 1989. 


\section{DISCUSIÓN}

En la actualidad Septo y Tabique son considerados por la RAE sinónimos, y ambos términos son utilizados indistintamente en las ciencias morfológicas, contribuyendo a la problemática sobre el manejo de numerosas denominaciones atribuidas a una misma estructura (Grinberg, 1999; Duque Parra et al., 2002; Losardo et al., 2010, 2015; Pérez-Rojas et al.). Así, al revisar los términos que se muestran en la Tabla I, constatamos que la Sociedad Anatómica Española traduce el término Septum al español como Tabique. Sin embargo, y de acuerdo con las evidencias encontradas, Septum también se traduce como Septo (Tabla II); lo que, en primera instancia, constituye una falta a los acuerdos realizados en el Congreso de Tokio el año 1975 respecto a la asignación de dos términos a una sola estructura, y refuerza la confusión del término Septum en su traducción al español como Tabique o Septo. De acuerdo con estos hallazgos encontrados, se demuestra entonces, una clara inconsistencia en la traducción de Septum hacia el español, y en el uso de términos de raíz latina para nombrar estructuras anatómicas en este idioma.

Es posible constatar en primera instancia, que la palabra Tabique es un término que deriva del árabe hispánico tasbík, por lo cual entre Tabique y Septum no existe realmente relación etimológica. En cambio, Septo corresponde a una palabra que deriva de manera directa del latín Septum evidenciando una estrecha relación entre el término latino original y su traducción hispánica. Las evidencias muestran que, en un principio, los términos Septo y Tabique eran utilizados en ámbitos totalmente distintos: Tabique se relaciona de manera predominante con la albañilería; mientras que, Septo, se asocia preferentemente con la anatomía de los seres del reino animal.

Es más, respecto al término Septo se evidencia, desde que existen antecedentes, se lo define como una división completa o incompleta de algo y que posteriormente se relaciona de manera exclusiva con las ciencias morfológicas; mientras que, Tabique es reconocido como el límite que separa dos ambientes físicos de construcción, en la albañilería. Sin embargo, se evidencia que a partir del año 1895 el término Tabique sí es relacionado con las ciencias anatómicas por el Diccionario Zerolo (Fig. 6), el cual sienta un precedente a la futura homologación del término. Posterior a esto, en el año 1917 en el Diccionario Alemany y Bolufer (Fig. 13), y en el diccionario Academia Usual de los años 1984 y 1989 (Figs. 12 y 14), Tabique ya se ocupa para definir la palabra Septo homologando los términos. De esto, podemos decir que aunque la utilización de ambos conceptos son considerados ya sinónimos absolutos para el español, y su uso es pertinente indistintamente en el uso coloquial del lenguaje, no cumplen de igual forma con las indicaciones dadas por la TAI, puesto que Tabique proviene de un término de raíz árabe mientras que Septo proviene de un término de raíz latina. Por tanto, su aplicación indistinta en la TAI es, al menos, confusa para las ciencias morfológicas.

Se demuestra aquí, que el término Tabique no es la mejor opción para nombrar los términos cuyo nombre taxonómico proviene de Septum; y, sugerimos cambiar la traducción de Septum a Septo, en reemplazo de Tabique; y, eliminar, además, la doble traducción expuesta en la tabla II dejando solo la traducción de Septum a Septo en la TAI.

PÉREZ-ROJAS, F.; CANALES-GUZMÁN, L. A.; CHIRINO-LETElier, C. \& PUEBLA-WUTH, R. S. Septum or "tabique"? Historical, etymological and proposal analysis for international anatomical terminology (TAI). Int. J. Morphol., 37(2):766-772, 2019.

SUMMARY: The growing interest in reconciling a common anatomical terminology makes it imperative to establish a universal language over time, and focus this objective on a systematic work, which allows to properly define and name the structures that make up the human body (Álvarez Guisbert \& Campohermoso Rodríguez, 2007). In the morphological sciences, the TAI establishes that the terms to name anatomical structures, use Latin and common terms of the Spanish language derived from a Latin root as the official language. In this work it is noted that a series of anatomical structures that in Spanish have been assigned with the name «tabique», do not correspond etymologically with the Latin root of the word with which they were originally named: Septum. We show that the word Septum is translated into Spanish literally as a septum, while the word «tabique» comes from a root of Arabic origin tasbík; and, therefore, it is not a term that complies with the TAI guidelines. Here we realize, according to an etymological and historical analysis of both words, that these became synonyms over time in the Spanish language. Even so, respecting TAI, the term «tabique» is not appropriate since its linguistic root is Arabic; and, therefore, we suggest changing the translation of Septum by «septo» into the anatomical structures reported in this research.

KEY WORDS: Terminologia Anatomica; Septum. 


\section{REFERENCIAS BIBLIOGRÁFICAS}

Álvarez Guisbert, O. \& Campohermoso Rodríguez, O. Evolución histórica conceptual de la Terminología Anatómica. Rev. Cuad., 52(1):113-7, 2007.

Anders, V. Etimología de Septo. Diccionario Etimológico Español en Línea, 2019. Disponible en: http://etimologias.dechile.net/?septo

Bluteau, R. Diccionario Castellano y Portuguez para facilitar a los castellanos el uso del Vocabulario Portuguez, y Latino, Vocabulário Portuguez e Latino, VIII. Lisboa Occidental, Na Officina de Pascoal da Silva, 1721.

Cabrera, R.; Ayeguí, J. P. \& Gil de Lara, J. D. Diccionario de Etimologías de la Lengua Castellana. Madrid, Impr. de M. Calero, 1837.

Comité Federal sobre Terminología Anatómica \& Sociedad Anatómica Española. Terminología Anatómica. Terminología Anatómica Internacional. Madrid, Médica Panamericana, 2001.

Duque Parra, J. E.; Gómez Arias, N. C. \& Giraldo R., D. P. Nomenclatura Anatómica Internacional. Med. U. P. B., 21(1):43-55, 2002.

Federative International Programme on Anatomical Terminologies. Terminologia Anatomica. International Anatomical Terminology. $2^{\mathrm{a}} \mathrm{ed}$. Stuttgart, Thieme, 2011.

García de Cortázar, F. Atlas de Historia de España. $2^{\mathrm{a}}$ ed. Barcelona, Planeta, 2005.

Grinberg, M. Epônimos em Cardiologia. Homenagem e exemplo. São Paulo, Livraria Roca, 1999.

Grkovic, I.; Marinovic Guic, M.; Kosta, V.; Poljicanin, A.; Caric, A. \& Vilovic, K. Designing anatomy program in modern medical curriculum: matter of balance. Croat. Med. J., 50(1):49-54, 2009.

Losardo, R. J.; Cruz Gutiérrez, R.; Rodríguez Torres, A.; Prates, J. C. \& Prates, N. E. V. B. Iberia-Latin-American Symposia of Morphological Terminology (SILAT). First two years and statute. Int. J. Morphol., 28(4):1323-6, 2010

Losardo, R. J.; Prates, N. E. V. B.; Arteaga-Martínez, M.; Cabral, R. H., García-Peláez, M. I. International Morphological Terminology: More than anatomy, histology and embryology. Int. J. Morphol., 33(1):4007, 2015.

Moya, M. P.; Contreras, M. \& del Sol, M. Lexema phy-sis fúsiV in Terminologia Anatomica. Int. J. Morphol., 35(3):919-24, 2017.

Pérez-Rojas, F.; Bustamante-Aliste, P. \& Puebla-Wuth, R. S. Tunic or Coat? Historical, etymological analysis and proposal for the International Anatomical Terminology. Int. J. Morphol., 36(1):284-9, 2018.

Resnick, M. C. Introducción a la Historia de la Lengua Española. Washington D. C., Georgetown University Press, 1981.

Universidad de Salamanca. Diccionario Médico-Biológico, Histórico y Etimológico. Salamanca, Universidad de Salamanca, 2018. Disponible en: https://dicciomed.usal.es/

Vargas, C. A.; Ottone, N. E.; Contreras, M. \& del Sol, M. Facies or Impressio in the Spleen? Int. J. Morphol., 34(3):1002-8, 2016.

Whitmore, I. Terminologia anatomica: new terminology for the new anatomist. Anat. Rec., 257(2):50-3, 1999.
Dirección para correspondencia:

Francisco Perez Rojas

Escuela de Medicina

Universidad Aut.noma de Chile

5 Poniente 1670

Talca

CHILE

Email: fperezr@uautonoma.cl

Recibido : 24-08-2018

Aceptado: 30-11-2018 